\title{
Exercício físico outdoor e indoor, bem-estar subjetivo e conexão com a Natureza: uma revisão sistemática
}

\section{Humberto da Nóbrega Alves', Gilivã Antônio Fridich', Thiago Siqueira Paiva de Souza ${ }^{1}$, Luiz Carlos Serrano López $^{2}$ e Reinaldo Farias Paiva de Lucena ${ }^{1}$}

${ }^{1}$ Universidade Federal da Paraíba. Centro de Ciências Exatas e da Natureza. Programa de Pós-Graduação em Desenvolvimento e Meio Ambiente. Campus I. João Pessoa-PB, Brasil (CEP 58051-900).E-mail: humbertonobrega@yahoo.com.br.

${ }^{2}$ Centro de Ciências da Saúde. Programa de Pós-Graduação em Neurociência Cognitiva. Campus I. João Pessoa-PB (CEP 58051-900).

Resumo. 0 presente estudo sintetiza as evidências disponíveis na literatura sobre a relação entre exercício físico outdoor e indoor, conexão com o meio ambiente e bem-estar a partir de busca sistemática no portal "periódicos capes" e "biblioteca virtual de saúde", utilizando os descritores relacionados ao tema. Foram selecionados treze artigos para leitura, análise e discussão. Os resultados mostraram que os exercícios outdoor apresentam efeitos estatisticamente significativos na melhoria e ganhos em aspectos psicoemocionais e aspectos relacionados à conectividade com a Natureza, no entanto em termos fisiológicos os resultados comparativos entre exercício outdoor e indoor não mostraram diferenças significativas entre essas duas categorias de exercícios.

Palavras-chaves: Exercício; Qualidade de vida; Revisão por pares; Saúde.

\begin{abstract}
Physical exercise outdoor and indoor, subjective well-being and connectedness to nature: A systematic review. The present study summarizes the evidence available in the literature on the relationship between outdoor and indoor exercise, connection with the environment and well-being from a systematic search on the portal "periodic capes" and "virtual health library", using the descriptors related to the theme. Thirteen articles were selected for reading, analysis and discussion. The results showed that outdoor exercises have statistically significant effects on improvement and gains in psycho-emotional aspects and aspects related to nature connectivity. However, in physiological terms the comparative results between outdoor and indoor exercise showed no significant differences between these two exercise categories.
\end{abstract}

Keywords: Exercise; Quality of life; Peer review; Health.
Recebido: 26/05/2019

Aceito

20/08/2019

Publicado:

30/04/2019

Acesso aberto

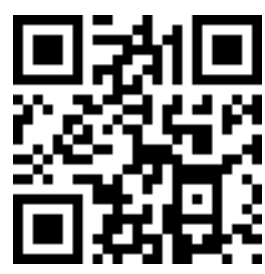

ORCID

(ㄷ) 0000-0002-3081-537X Humberto da Nóbrega Alves

(ㄱ) 0000-0003-1057-5134 Gilivã Antônio Fridich

D 0000-0002-5113-8566 Thiago Siqueira Paiva de Souza

D 0000-0002-9983-5563

Luiz Carlos Serrano López

(D) 0000-0003-4775-7775

Reinaldo Farias Paiva de Lucena 


\section{Introdução}

0 exercício físico é um importante meio de prevenção e promoção de saúde e qualidade de vida da população, sendo que a prática regular tem sido associada diretamente à melhoria no bem-estar, além de também estar associada à adoção de outras condutas relacionadas à saúde da população (Nahas, 1997; Lopes e Alterthum, 1999; Minayo et al., 2000; Carvalho, 2001; Piko e Keresztes, 2006; Timperio et al., 2006; Haskell et al., 2007). Podendo-se constatar além destes benefícios já relatados e comprovados cientificamente também outros benefícios específicos como os mentais, emocionais (Marselle et al., 2014), abrindo um vasto campo de estudos para novas pesquisas nesta linha de estudo.

0 bem-estar é considerado como um dos conceitos chaves da saúde, sendo trabalhado na vertente do bem-estar subjetivo, bem-estar psicológico e bemestar no trabalho (Siqueira et al., 2008), entendido como a integração harmoniosa entre os componentes mentais, físicos, espirituais e emocionais (Nahas et al., 2000), sendo conceitos bastante estudados na literatura científica no campo de conhecimento da saúde e qualidade de vida, que evidenciam uma estreita relação entre estilo de vida e saúde, considerando as características nutricionais, nível de estresse, ambientais e atividades físicas habituais (Blair, 1993; Gill e Feinstein, 1994; Ryff e Keyes, 1995; Diener et al., 1997; Diener et al., 1999; Diener et al., 2003; Seligman e Csikszentmihalyi, 2000; Albuquerque e Tróccoli, 2004), além dos benefícios e melhorias advindos de treinamentos com exercício físicos regulares em diversas populações de jovens, adultos e idosos (Browne et al., 1994; Bowling, 1995; Farquar et al., 1995; Fleck, 2000; Freitas et al., 2007).

Estudos recentes envolvendo exercício físico, bem-estar e conexão com a natureza (Piko e Keresztes, 2006;
Timperio et al., 2006; Lopes e Alterthum, 1999; Diener et al.,2003) tem apontado também para ganhos importantes tanto no bem-estar subjetivo (Ryff e Keyes, 1995; Diener et al., 1997; Diener et al., 1999; Nahas et al., 2000; Seligman e Csikszentmihalyi, 2000; Diener et al., 2003; Albuquerque e Tróccoli, 2004; Siqueira e Padovan, 2008) quanto em indicadores de saúde/qualidade de vida tais como Índice de Massa Corporal, Relação Cintura Quadril, percentual de gordura, etc. (Must et al., 1991; Anjos, 1992; Abrantes et al., 2003) e em uma maior conectividade com o meio ambiente (Bruhns, 1997; Milanezi et al., 1997; Tahara et al., 2006).

Em se tratando de investigações científicas envolvendo exercício físico e conexão com a natureza as pesquisas tem apontado para inter-relações entre estas variáveis, destacando a questão de uma maior percepção positiva da conservação e preservação ambiental e promoção de atitudes pro ambientais, tanto em praticantes de exercício indoors (ambientes fechados), como praticantes de exercício outdoor (Marinho, 2001; Chao, 2004; Marinho e Inácio, 2007; Florindo et al., 2011; Silva et al., 2014), indicando melhorias na saúde $\mathrm{e}$ qualidade de vida com incorporação de hábitos saudáveis associando exercício físico, alimentação adequada e maior conectividade ambiental (Minayo et al., 2000; Carvalho, 2001; Marselle et al., 2014).

O objetivo desta revisão sistemática é sintetizar as evidências disponíveis na literatura sobre estudos que tratem da relação entre o exercício físico outdoor/exercício físico indoor, conexão com a natureza e bem-estar subjetivo com foco voltados para a melhoria da qualidade de vida e saúde da população.

\section{Materiais e métodos}

Optou-se pela revisão sistemática como metodologia de pesquisa, por 
entender que a mesma se configura com uma forma de pesquisa criteriosa que utiliza como fonte de dados a literatura sobre temas específicos mediante a aplicação de métodos explícitos e sistematizados de busca, apreciação crítica e síntese de informação selecionada (Sampaio e Mancine, 2007).

\section{Estratégia de busca e elegibilidade}

O presente estudo seguiu os critérios metodológicos estabelecidos pelo Preferred Reporting Items for Systematic Reviews and MetaAnalysis/PRISMA (Galvão et al., 2015). Realizou-se ampla e exaustiva revisão da literatura buscando descritores que atendessem aos objetivos do estudo. Para estratégia de busca sistemática nesta pesquisa, optou-se por utilizar os seguintes descritores: "outdoor exercise" OR "indoor exercise" AND "well-being" OR "connectedness to nature" (em inglês) e "exercício outdoor" OR "exercício indoor" AND "bem-estar" OR "conexão com a natureza" (em português), além de combinações entre os descritores isoladamente também em inglês e em português usando os operadores lógicos disponíveis nas ferramentas de busca.

A busca foi realizada no portal "Periódicos Capes" sem delimitação de período de tempo dos artigos encontrados, sendo utilizadas as seguintes bases bibliográficas: OneFile (GALE), Scopus (Elsevier), MEDLINE/ PubMed (NLM), Science Citation Index Expanded (Web of Science), Social Sciences Citation Index (Web of Science), PMC (PubMed Central), Cambridge Journals (Cambridge University Press), Directory of Open Access Journals (DOAJ), JSTOR Archival Journals, Sociological Abstracts, Wiley (CrossRef), Wiley Online Library, Pollution Abstracts, Science Direct Journals (Elsevier), BioMed Central, Duke University Press (CrossRef), Duke University Press Journals Online, CABI Publishing
(CrossRef), Arts \& Humanities Citation Index (Web of Science) e Wolters Kluwer - Ovid - Lippincott Williams \& Wilkins (CrossRef) e a Biblioteca Virtual de Saúde/Brasil.

\section{Critérios de inclusão e exclusão}

Para a inclusão dos artigos, foram estabelecidos os seguintes critérios: a) estudos originais; b) Estudos que tratem da relação entre exercício físico outdoor/exercício físico indoor, conexão com a natureza e bem-estar subjetivo, com foco na qualidade de vida e saúde da população de forma local ou global; c) Estudos com amostra representativa de alguma população definida. Não foram incluídos artigos sem relação com o tema, artigos de revisão e estudos de validação.

\section{Extração dos dados}

0 processo de revisão sistemática foi realizada em três fases. A primeira fase consistiu na leitura dos títulos; a segunda fase consistiu na leitura dos resumos; a terceira fase na leitura completa dos textos e organização do quadro dos artigos sendo observados os seguintes aspectos: a) autores do artigo; b) ano de publicação; c) localidade da pesquisa; d) amostra; e) faixa etária; f) instrumentos de pesquisa/forma de aplicação; g) principais resultados da pesquisa. Não foram incluídos artigos sem relação com o tema, artigos de revisão e estudos de validação.

Todas as publicações foram analisadas por quatro pesquisadores, sendo que cada um elaborou um quadro com a síntese das informações extraídas dos artigos e em seguida, os quadros foram comparados a fim se verificar a concordância entre os pares, e na eventualidade de divergências entre informações sintetizadas pelos pares, o artigo foi revisado por um quinto membro da equipe a fim de identificar as incoerências e definir quais as evidências que $\mathrm{o}$ artigo efetivamente veiculava. 


\section{Resultados}

Após as buscas e leitura dos títulos e resumos das publicações nas bases bibliográficas do portal "periódicos capes" referenciada na metodologia do presente artigo de revisão, foram analisados na primeira fase no banco de dados um total de 1.560, sendo 70 artigos incluídos inicialmente; na segunda fase de leitura dos títulos foram analisados 70 artigos e incluídos 42 artigos; na terceira fase de leitura de resumos foram analisados 42 artigos e incluídos 35 artigos; na quarta e última fase de leitura dos textos completos foram analisados 35 artigos e incluídos 13 artigos (Figura 1).
Estes artigos selecionados nesta revisão sistemática foram analisados e aprovados pelo grupo de revisores que extraíram seus dados para discussão e reflexão sobre a relação entre a exercício físico outdoor/exercício físico indoor, conexão com a natureza e bem-estar subjetivo com foco voltados para a melhoria da qualidade de vida e saúde da população.

Em cada uma das fases os artigos que foram excluídos não estavam de acordo com os critérios de inclusão e exclusão desta revisão sistemática, referenciados na metodologia deste artigo.

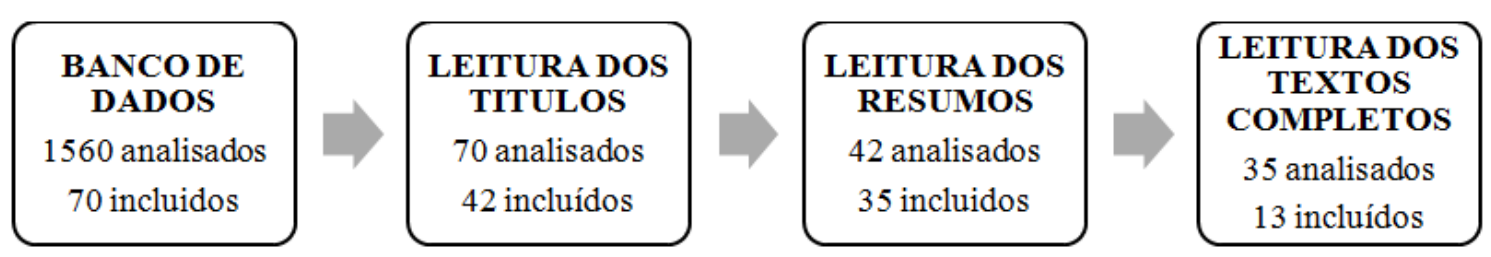

Figura 1. Fluxograma do resultado do processo de seleção dos artigos da revisão sistemática

Na Figura 1 do fluxograma do resultado do processo de seleção dos artigos da revisão sistemática pode-se constatar um número significativo de estudos envolvendo os temas exercício outdoor, exercício indoor, bem-estar e conexão com a natureza (1560 artigos), no entanto após leitura dos títulos, dos resumos e dos textos completos apenas 13 estudos (Matsouka et al., 2005; Loureiro e Veloso, 2014; Pasanen et al., 2014; Puett et al., 2014; Weng e Chiang, 2014;Calogiuri et al., 2015; Wood et al., 2015;Rogerson et al., 2016; LacharitéLemieux e Dionne, 2016; Krinski et al., 2017; Niedermeier et al., 2017; Fuegen e Breitenbecher, 2018; Legrand et al., 2018) que buscam analisar a relação comparativa entre o exercício outdoor e indoor com o bem-estar e conexão com a natureza com foco na qualidade de vida e saúde da população.

Na tabela 1, são apresentados os detalhes e informações dos 13 estudos (Matsouka et al., 2005; Loureiro e Veloso, 2014; Pasanen et al., 2014; Puett et al., 2014; Weng e Chiang, 2014;Calogiuri et al., 2015; Wood et al., 2015; Rogerson et al., 2016; Lacharité-Lemieux e Dionne, 2016; Krinski et al., 2017; Niedermeier et al., 2017; Fuegen e Breitenbecher, 2018; Legrand et al., 2018) inclusos na revisão. Foram avaliados: ano de publicação, número de sujeitos, número de sujeitos por sexo, idade e instrumentos de coleta de dados das pesquisas realizadas.

Os treze estudos (Matsouka et al., 2005; Loureiro e Veloso, 2014; Pasanen et al., 2014; Puett et al., 2014; Weng e Chiang, 2014;Calogiuri et al., 2015; Wood 
et al., 2015;Rogerson et al., 2016; Lacharité-Lemieux e Dionne, 2016; Krinski et al., 2017; Niedermeier et al., 2017; Fuegen e Breitenbecher, 2018; Legrand et al., 2018) inclusos na revisão, 12 estudos, 92\% (Loureiro e Veloso, 2014; Pasanen et al., 2014; Puett et al., 2014; Weng e Chiang, 2014; Wood et al., 2016; Calogiuri et al., 2015 ; Rogerson et al., 2016; Lacharité-Lemieux e Dionne, 2016; Krinski et al., 2017; Niedermeier et al., 2017; Fuegen e Breitenbecher, 2018; Legrand et al., 2018) sobre a relação entre exercício indoor, outdoor, bemestar subjetivo e conexão com a natureza produzidas em sua maioria nos últimos 05 anos, sendo destes, quatro estudos realizados em 2014 (Loureiro e Veloso, 2014; Pasanen et al., 2014; Puett et al., 2014; Weng e Chiang, 2014), dois estudos em 2015 (Calogiuri et al., 2015; Wood et al., 2015), dois estudos em 2016 (Rogerson et al., 2016; LacharitéLemieux e Dionne, 2016), dois estudos em 2017(Krinski et al., 2017; Niedermeier et al., 2017), dois estudos em 2018 (Fuegen e Breitenbecher, 2018; Legrand et al., 2018) e apenas um estudo realizado no ano de em 2005, 8\% (Matsouka et al., 2005) como pode-se visualizar na Tabela 1, indicando assim um interesse recente em investigações que envolvem a temática em questão.

Tabela 1. Características de ano, localidade, amostra, sexo, idade e instrumentos de pesquisa dos sujeitos descritos nas pesquisas da revisão sistemática.

\begin{tabular}{|c|c|c|c|c|c|c|c|}
\hline Autores & Ano & Localidade & $\mathbf{N}$ & $\hat{0}$ & 온 & Idade & Instrumento de pesquisa \\
\hline $\begin{array}{l}\text { Matsouka et al. } \\
(2005)\end{array}$ & 2005 & $\begin{array}{l}\text { Komotini, } \\
\text { Grécia }\end{array}$ & 55 & - & 55 & $60-75$ & $\begin{array}{l}\text { Inventário de afetos com } 12 \\
\text { exercícios induzidos, avaliando } \\
\text { ações positivas, revitalização, } \\
\text { tranquilidade e exaustão física. }\end{array}$ \\
\hline $\begin{array}{l}\text { Loureiro e } \\
\text { Veloso (2014) }\end{array}$ & 2014 & $\begin{array}{l}\text { Lisboa, } \\
\text { Portugal }\end{array}$ & 282 & 159 & 123 & $32-33$ & $\begin{array}{l}\text { Escala de Conexão Com a } \\
\text { Natureza (CNS); Escala de } \\
\text { Afetos positivos e negativos e } \\
\text { positivos (PANAS); Escala de } \\
\text { Experiência Subjetiva com } \\
\text { Exercício (SEES) }\end{array}$ \\
\hline $\begin{array}{l}\text { Weng e Chiang } \\
(2014)\end{array}$ & 2014 & $\begin{array}{l}\text { Chiayi, } \\
\text { Taiwan }\end{array}$ & 203 & 100 & 203 & $18-26$ & $\begin{array}{l}\text { Teste de controle padrão cubo } \\
\text { Necker - NCPC (atenção); } \\
\text { Inventário do estado de } \\
\text { ansiedade- STAI (ansiedade) }\end{array}$ \\
\hline $\begin{array}{l}\text { Pasanen, et al. } \\
(2014)\end{array}$ & 2014 & $\begin{array}{l}\text { Tampere, } \\
\text { Finlândia }\end{array}$ & 2.070 & 1.159 & 911 & $15-74$ & $\begin{array}{l}\text { Sub escala de bem-estar } \\
\text { emocional; Escala de avaliação } \\
\text { do sono; Avaliação da pressão } \\
\text { arterial (atividade física) }\end{array}$ \\
\hline $\begin{array}{l}\text { Puett et al. } \\
(2014)\end{array}$ & 2014 & Dallas, EUA & 11.649 & 8.657 & 2.992 & 46 & $\begin{array}{l}\text { Questionário e avaliação clínica } \\
\text { da relação da atividade física no } \\
\text { meio ambiente (exame físico } \\
\text { exercício teste de exercício } \\
\text { máximo na esteira, análise } \\
\text { química do sangue, antropo- } \\
\text { metria, tensão, estresse, } \\
\text { percepção emocional, saúde e } \\
\text { dados socioeconômicos) - estu- } \\
\text { do prospectivo }\end{array}$ \\
\hline $\begin{array}{l}\text { Wood et al. } \\
(2015)\end{array}$ & 2015 & $\begin{array}{l}\text { Inglaterra, } \\
\text { Manchester }\end{array}$ & 269 & 152 & 117 & 56 & $\begin{array}{l}\text { Questionários de auto estima } \\
\text { (Rosenberg scale); humor } \\
\text { (POMS); saúde geral (GHQ-12) e } \\
\text { distúrbio do humor antes e } \\
\text { após sessão. Tempo gasto na } \\
\text { semana em jardinagens. IMC. }\end{array}$ \\
\hline
\end{tabular}


Tabela 1. Continuação.

\begin{tabular}{|c|c|c|c|c|c|c|c|}
\hline Autores & Ano & Localidade & $\mathbf{N}$ & $\hat{0}$ & 우 & Idade & Instrumento de pesquisa \\
\hline $\begin{array}{l}\text { Calogiuri et al. } \\
(2015)\end{array}$ & 2015 & $\begin{array}{l}\text { Noruega, } \\
\text { Troms }\end{array}$ & 14 & 7 & 7 & 49 & $\begin{array}{l}\text { Escala de percepção de esforço } \\
\text { - Escala de Borg 20-item; } \\
\text { Restauração percebida do meio } \\
\text { ambiente; Escala de restauração } \\
\text { percebida do meio ambiente } \\
\text { (Hartig, 1997); Satisfação - } \\
\text { pergunta única sobre a } \\
\text { satisfação.; Afetos - PANAS; } \\
\text { Intenções de se exercitar no } \\
\text { futuro; Escala de três } \\
\text { itens/verificar comportamento } \\
\text { do indivíduo relacionado ao } \\
\text { exercício nas próximas 10 } \\
\text { semanas; Comportamento } \\
\text { relacionado ao exercício - } \\
\text { LTEQ; Aptidão Física - Teste de } \\
\text { rampa para análise de VO2 } \\
\text { máx.; Conectividade com a } \\
\text { natureza - Escala Mayer e } \\
\text { Frantz (2004); Estudo } \\
\text { qualitativo (meio ambiente e } \\
\text { influência nos sentimentos. }\end{array}$ \\
\hline $\begin{array}{l}\text { Rogerson et al. } \\
(2016)\end{array}$ & 2016 & $\begin{array}{l}\text { Colchester, } \\
\text { Inglaterra }\end{array}$ & 24 & 5 & 19 & $18-75$ & $\begin{array}{l}\text { Questionário Pré e pós exercício } \\
\text { (atenção dirigida, humor, tempo } \\
\text { de interação social, esforço } \\
\text { percebido, intenção futura para } \\
\text { comportamento com exercício e } \\
\text { sessão de exercício em } \\
\text { repouso). }\end{array}$ \\
\hline $\begin{array}{l}\text { Lacharité- } \\
\text { Lemieux e } \\
\text { Dionne (2016) }\end{array}$ & 2016 & $\begin{array}{l}\text { Québec, } \\
\text { Canadá }\end{array}$ & 23 & - & 23 & 61 & $\begin{array}{l}\text { Escala de atividades físicas para } \\
\text { idosos (resistência, força } \\
\text { muscular, aderência e nível de } \\
\text { atividade física.; Teste de } \\
\text { caminhada } \\
\text { submáxima; graduada } \\
\text { antropométricas e Medidas } \\
\text { composição corporal; Medidas } \\
\text { de pressão sanguínea/ } \\
\text { frequência cardíaca, VO2max, } \\
\text { glicose em jejum, insulina e } \\
\text { lipídeos plasmáticos. }\end{array}$ \\
\hline $\begin{array}{l}\text { Krinski et al. } \\
\text { (2017) }\end{array}$ & 2017 & $\begin{array}{c}\text { Petrolina/ } \\
\text { Londrina/ } \\
\text { Natal, } \\
\text { Brasil. }\end{array}$ & 38 & - & 38 & $45-46$ & $\begin{array}{l}\text { Avaliação da composição } \\
\text { corporal (peso, altura, IMC); } \\
\text { Teste de caminhada graduada } \\
\text { máxima (velocidade, consumo } \\
\text { de oxigênio e frequência } \\
\text { cardíaca-VO2max, VO2vt e } \\
\text { HRmax); Avaliação psicológica } \\
\text { (resposta afetiva, percepção } \\
\text { subjetiva do esforço, escala de } \\
\text { sensação sentida, foco da } \\
\text { atenção, apreciação e intenção } \\
\text { para caminhar no futuro }\end{array}$ \\
\hline $\begin{array}{l}\text { Niedermeier et } \\
\text { al (2017) }\end{array}$ & 2017 & $\begin{array}{c}\text { Innsbruck, } \\
\text { Áustria }\end{array}$ & 42 & 22 & 20 & $18-70$ & $\begin{array}{l}\text { Escala de sentimentos (Feeling } \\
\text { Scale); Escala de excitação (Felt } \\
\text { Arousal Scale); Escala de } \\
\text { pesquisa do humor (Mood } \\
\text { Survey Scale) }\end{array}$ \\
\hline
\end{tabular}


Tabela 1. Continuação.

\begin{tabular}{|c|c|c|c|c|c|c|c|}
\hline Autores & Ano & Localidade & $\mathbf{N}$ & $\hat{\sigma}$ & 우 & Idade & Instrumento de pesquisa \\
\hline $\begin{array}{l}\text { Fuegen e } \\
\text { Breitenbecher } \\
(2018)\end{array}$ & 2018 & $\begin{array}{l}\text { Highland } \\
\text { Heights, } \\
\text { EUA }\end{array}$ & 181 & 73 & 108 & $17-75$ & $\begin{array}{l}\text { Sequência de inversão digital } \\
\text { (Digit Span Backward); Teste } \\
\text { das modalidades de símbolos } \\
\text { digitais (Symbol Digit } \\
\text { Modalities Test); Escala de } \\
\text { afetos positivos e negativos } \\
\text { (Positive and Negative Affect } \\
\text { Schedule); Listagem adjetiva de } \\
\text { ativação-desativação } \\
\text { (Activation-Deactivation } \\
\text { Adjective Checklist): Clima }\end{array}$ \\
\hline & & & & & & & $\begin{array}{l}\text { Adjective } \\
\text { (Weather) }\end{array}$ \\
\hline $\begin{array}{l}\text { Legrand, et al. } \\
\text { (2018) }\end{array}$ & 2018 & $\begin{array}{l}\text { Reims, } \\
\text { França }\end{array}$ & 18 & 06 & 12 & 26 & $\begin{array}{l}\text { Inventário de sinais } \\
\text { depressivos; Perfil do estado de } \\
\text { humor (energia e fadiga) }\end{array}$ \\
\hline
\end{tabular}

Legenda: N (amostra populacional), $\widehat{\partial}$ (masculino),

A somatória total da população investigada nos estudos foi de 14.868 participantes, sendo 10.340 homens (70\%) e 4.528 mulheres (30\%), com idade entre 15-75 anos. Destes estudos apenas três trabalhos apresentaram uma amostra somente de mulheres (Matsouka et al., 2005; Lacharité-Lemieux e Dionne, 2016; Krinski et al., 2017) e nove estudos apresentaram uma amostra mista constituída de homens e de mulheres (Loureiro e Veloso, 2014; Pasanen et al., 2014; Puett et al., 2014; Calogiuri et al., 2015; Wood et al., 2015; Rogerson et al., 2016; Niedermeier et al., 2017; Fuegen e Breitenbecher, 2018; Legrand et al., 2018) e nenhum estudo apresentou amostra somente constituída de homens.

Também é importante um olhar atento sobre as metodologias adotadas por estas pesquisas, trabalhando com questionários de afetos, conexão com a natureza, bem-estar psicológico e percepção (Matsouka et al., 2005; Loureiro e Veloso, 2014; Pasanen et al., 2014; Puett et al., 2014; Weng e Chiang, 2014;Calogiuri et al., 2015; Wood et al., 2015;Rogerson et al., 2016; LacharitéLemieux e Dionne, 2016; Krinski et al., 2017; Niedermeier et al., 2017; Fuegen e Breitenbecher, 2018; Legrand et al., 2018) associando também com coleta dos dados de composição corporal, antropométrico e fisiológico realizado com metodologias de coleta de dados bastante utilizados na área de saúde como antropometria, análises bioquímicas e fisiológica (Pasanen et al., 2014; Puett et al., 2014; Calogiuri et al., 2015;Wood et al., 2015; LacharitéLemieux e Dionne, 2016; Krinski et al., 2017).

Outro aspecto a ser destacados são as localidades que os estudos foram desenvolvidos, sendo que a maioria dos estudos nesta temática foram feitos na Europa (Matsouka et al., 2005; Loureiro e Veloso, 2014; Puett et al., 2014; Calogiuri et al., 2015; Wood et al., 2015;Rogerson et al., 2016;Niedermeier et al., 2017; Legrand et al., 2018), em seguida dois estudos realizados nos Estados Unidos (Weng e Chiang, 2014; Fuegen e Breitenbecher, 2018), um estudo na Ásia (Pasanen et al., 2014), outro estudo no Canadá (Lacharité-Lemieux e Dionne, 2016) e um estudo no Brasil (Krinski et al., 2017). Esta constatação permite concluir que existe uma grande necessidade de investigações com este foco de estudo no Brasil, uma vez que a maioria dos estudos foram desenvolvidos na Europa, Ásia e América do Norte, demonstrando assim uma necessidade de estudos nesta linha temática em zonas 
climáticas diferentes da Zona Temperada do Norte.

Na Tabela 2 são apresentados os detalhes dos conteúdos e aspectos observados nos estudos da revisão sistemática, bem como seus principais resultados, sendo observado que em todos os estudos (Matsouka et al., 2005; Loureiro e Veloso, 2014; Pasanen et al., 2014; Puett et al., 2014; Weng e Chiang,
2014; Calogiuri et al., 2015; Wood et al., 2015;Rogerson et al., 2016; LacharitéLemieux e Dionne, 2016; Krinski et al., 2017; Niedermeier et al., 2017; Fuegen e Breitenbecher, 2018; Legrand et al., 2018) apareceram elementos que influenciam direta ou indiretamente no bem-estar e conectividade com a natureza diante da prática de exercício indoor e outdoor.

Tabela 2. Conteúdos abordados e os principais resultados da revisão sistemática.

\begin{tabular}{|c|c|c|c|}
\hline Autores & Bem-estar & $\begin{array}{c}\text { Conectividade com } \\
\text { o meio ambiente } \\
\end{array}$ & Outros resultados \\
\hline $\begin{array}{l}\text { Matsouka } \\
\text { et al. } \\
(2005)\end{array}$ & $\begin{array}{l}\uparrow \text { Bem-estar psicológico. } \\
\text { Indoor = outdoor }\end{array}$ & $\begin{array}{lr}\text { Estados } & \text { de } \\
\text { sentimentos } & \\
\text { possuem associação } \\
\text { entre } & \text { as } \\
\text { propriedades } & \text { de } \\
\text { estimulo } & \text { ao } \\
\text { exercício ao ar livre. }\end{array}$ & $\begin{array}{l}\uparrow \text { Afetos positivos, ações positivas, } \\
\text { revitalização, tranquilidade } \\
\downarrow \text { Afetos negativos, exaustão física }\end{array}$ \\
\hline $\begin{array}{l}\text { Loureiro e } \\
\text { Veloso } \\
(2014) \\
\end{array}$ & $\begin{array}{l}\uparrow \text { Bem-estar associado } \\
\text { ao exercício } \\
\text { Outdoor }>\text { Indoor }\end{array}$ & $\begin{array}{l}\text { Conectividade é um } \\
\text { preditor do bem- } \\
\text { estar }\end{array}$ & $\uparrow$ emoções positivas \\
\hline $\begin{array}{l}\text { Weng e } \\
\text { Chiang } \\
(2014)\end{array}$ & $\begin{array}{l}\uparrow \text { Bem-estar associado } \\
\text { ao exercício } \\
\text { Outdoor }>\text { Indoor }\end{array}$ & $\begin{array}{l}\text { A caminhada foi a } \\
\text { melhor atividade ao } \\
\text { ar livre que aponta } \\
\text { para melhoria na } \\
\text { saúde mental. }\end{array}$ & $\begin{array}{l}\uparrow \text { Restauração da atenção } \\
\downarrow \text { ansiedade } \\
\text { Outdoor > Indoor }\end{array}$ \\
\hline $\begin{array}{l}\text { Pasanen } \\
\text { et al. } \\
(2014)\end{array}$ & $\begin{array}{l}\uparrow \text { Bem-estar emocional } \\
\text { Outdoor > Indoor }\end{array}$ & $\begin{array}{l}\text { Exercício ao ar livre } \\
\text { tem conexão com } \\
\text { bem } \\
\text { emocional. }\end{array}$ & $\begin{array}{l}\uparrow \text { Saúde (Outdoor = Indoor) } \\
\text { Atividades outdoor não promovem } \\
\text { efeitos sobre qualidade do sono. }\end{array}$ \\
\hline $\begin{array}{l}\text { Puett et } \\
\text { al. }(2014)\end{array}$ & $\begin{array}{l}\uparrow \text { Bem-estar associado } \\
\text { ao exercício } \\
\text { Outdoor > Indoor } \\
\text { em populações mais } \\
\text { ativas }\end{array}$ & $\begin{array}{l}\text { Exercício outdoor é } \\
\text { um fator protetivo } \\
\text { em relação a tensão, } \\
\text { controle de estresse, } \\
\text { melhora a saúde } \\
\text { emocional e geral. }\end{array}$ & $\begin{array}{l}\uparrow \text { controle do estresse e percepção } \\
\text { da saúde (maior associação em } \\
\text { atividades outdoor com populações } \\
\text { mais ativas); } \\
\text { Sugere associação entre sessões de } \\
\text { exercício outdoor e indoors para } \\
\text { melhorias na saúde. }\end{array}$ \\
\hline $\begin{array}{l}\text { Wood et } \\
\text { al. (2015) }\end{array}$ & $\begin{array}{l}\text { Atividades ao ar livre } \\
\text { promovem bem-estar } \\
\text { mental }\end{array}$ & $\begin{array}{l}\text { Atividades ao ar } \\
\text { livre promovem } \\
\text { bem-estar mental }\end{array}$ & $\begin{array}{l}\uparrow \text { Auto estima } \\
\downarrow \text { Distúrbios negativos no humor, } \\
\text { IMC }\end{array}$ \\
\hline $\begin{array}{l}\text { Calogiuri } \\
\text { et al. } \\
(2015)\end{array}$ & $\begin{array}{l}\uparrow \text { Bem-estar } \\
\text { Outdoor > Indoor }\end{array}$ & $\begin{array}{l}\text { Exercício ao ar livre } \\
\text { fornece mais } \\
\text { respostas afetivas, } \\
\text { percepção de } \\
\text { esforço, melhoria da } \\
\text { taxa cardíaca e da } \\
\text { satisfação. }\end{array}$ & $\begin{array}{l}\text { FC em repouso e PSE (outdoor = } \\
\text { indoor); } \\
\uparrow \text { Fascinação, satisfação, referências } \\
\text { positivas em diálogos (outdoor > } \\
\text { indoor) } \\
\text { Motivação - em exercícios indoor a } \\
\text { motivação provém das instruções e } \\
\text { informações; no outdoor parece que } \\
\text { o ambiente promove a motivação. }\end{array}$ \\
\hline
\end{tabular}


Tabela 2. Conteúdos abordados e os principais resultados da revisão sistemática.

\begin{tabular}{|c|c|c|c|}
\hline Autores & Bem-estar & $\begin{array}{l}\text { Conectividade com } \\
\text { o meio ambiente }\end{array}$ & Outros resultados \\
\hline $\begin{array}{l}\text { Rogerson } \\
\text { et al. } \\
(2016)\end{array}$ & $\begin{array}{l}\uparrow \text { Bem-estar } \\
\text { Outdoor > Indoor }\end{array}$ & $\begin{array}{l}\uparrow \quad \text { Atenção } \\
\text { direcionada, } \\
\text { interações sociais e } \\
\text { adesão (outdoor > } \\
\text { indoor) }\end{array}$ & $\begin{array}{l}\text { Humor e percepção de esforço } \\
\text { (indoor=outdoor) }\end{array}$ \\
\hline $\begin{array}{l}\text { Lacharité- } \\
\text { Lemieux e } \\
\text { Dionne } \\
(2016)\end{array}$ & $\begin{array}{lr}\text { Não } & \text { avaliado } \\
\text { diretamente, mas } \\
\text { indiretamente pelas } \\
\text { melhorias fisiológicas } \\
\text { de melhoria na saúde e } \\
\text { por sua vez no bem } \\
\text { estar da saúde. }\end{array}$ & $\begin{array}{l}\text { Maior conexão do } \\
\text { exercício outdoor } \\
\text { com aderência ao } \\
\text { exercício e exercício } \\
\text { indoor a melhorias } \\
\text { fisiológicas. }\end{array}$ & $\begin{array}{l}\uparrow \mathrm{VO}_{2 \max } \text { e composição corporal } \\
\text { (indoor > outdoor); } \\
\uparrow \text { Força corporal (Outdoor > Indoor) } \\
\downarrow \text { Pressão Arterial repouso } \\
\text { (Outdoor > indoor) }\end{array}$ \\
\hline $\begin{array}{l}\text { Krinski et } \\
\text { al. (2017) }\end{array}$ & $\begin{array}{l}\uparrow \text { Foco, atenção, afetos } \\
\text { positivos, adesão e } \\
\text { sensação de prazer } \\
\text { (Outdoor > Indoor) }\end{array}$ & \begin{tabular}{lr}
\multicolumn{2}{l}{ Exercício outdoor } \\
tem conexão com \\
aderência & ao \\
exercício. & \\
\end{tabular} & $\begin{array}{l}\uparrow \text { Velocidade, consumo de oxigênio } \\
\text { (Outdoor = Indoor) } \\
\mathrm{C} \text { Pressão arterial, Frequência } \\
\text { cardíaca, IMC (Outdoor = Indoor) }\end{array}$ \\
\hline $\begin{array}{l}\text { Niedermei } \\
\text { er et al. } \\
(2017)\end{array}$ & $\begin{array}{l}\uparrow \text { Bem-estar associado } \\
\text { ao exercício } \\
\text { Outdoor > Indoor, } \\
\text { aumento das valências } \\
\text { afetivas, ativação e } \\
\text { diminuição da fadiga } \\
\text { com exercício outdoor. }\end{array}$ & $\begin{array}{l}\text { Exercício outdoor } \\
\text { tem maior conexão } \\
\text { com melhorias } \\
\text { afetivas que o } \\
\text { exercício indoor. }\end{array}$ & $\begin{array}{l}\uparrow \text { Afetos, ativação, euforia, calma } \\
\text { (Outdoor > Indoor) } \\
\downarrow \text { Fadiga e ansiedade } \\
\text { (Outdoor > Indoor) }\end{array}$ \\
\hline $\begin{array}{l}\text { Fuegen e } \\
\text { Breitenbe } \\
\text { cher } \\
(2018)\end{array}$ & $\begin{array}{l}\uparrow \text { Bem-estar } \\
\text { Outdoor > Indoor } \\
\uparrow \text { Estados de humor, } \\
\text { energia, atenção } \\
\text { (Outdoor > Indoor) } \\
\end{array}$ & $\begin{array}{l}\text { Exercício ao ar livre } \\
\text { tem conexão com } \\
\text { bem-estar } \\
\text { emocional. (afetos } \\
\text { positivos) }\end{array}$ & $\downarrow$ Cansaço (Outdoor > Indoor) \\
\hline $\begin{array}{l}\text { Legrand } \\
\text { et al. } \\
(2018)\end{array}$ & $\begin{array}{l}\text { Não se observaram } \\
\text { diferenças significativas } \\
\text { entre exercício indoor e } \\
\text { outdoor. }\end{array}$ & $\begin{array}{l}\text { Não se observaram } \\
\text { diferenças } \\
\text { significativas entre } \\
\text { exercício indoor e } \\
\text { outdoor }\end{array}$ & $\uparrow$ Energia (Outdoor = Indoor) \\
\hline
\end{tabular}

Legenda: $\uparrow$ (aumento); $\downarrow$ (redução); > (maior que); = (igual); IMC (índice de massa corporal); PSE (percepção subjetiva de esforço); FC (frequência cardíaca); V02max (consumo máximo de oxigênio).

De acordo com os resultados obtidos nos estudos desta revisão sistemática (Matsouka et al., 2005; Loureiro e Veloso, 2014; Pasanen et al., 2014; Puett et al., 2014; Weng e Chiang, 2014;Calogiuri et al., 2015; Wood et al., 2015; Rogerson et al., 2016; LacharitéLemieux e Dionne, 2016; Krinski et al., 2017; Niedermeier et al., 2017; Fuegen e Breitenbecher, 2018; Legrand et al.,
2018) as investigações científicas apontam para melhorias no bem-estar e uma maior conectividade com a natureza com os praticantes de exercícios ao ar livre. Por sua vez, efeitos fisiológicos apareceram de forma bem similar em alguns estudos (Matsouka et al., 2005; Puett et al., 2014; Wood et al., 2015;Krinski et al., 2017; Legrand et al., 2018). 
Lacharité-Lemieux e Dionne (2016) mostram que os efeitos fisiológicos estavam mais associados aos treinamentos com exercício indoor, diferente de uma outra pesquisa feita por Niedermeier et al. (2017) que apontaram para uma diminuição da fadiga em treinamento com exercício outdoor e também observou-se em outro trabalho de Calogiuri et al. (2015) uma diminuição de IMC também em treinamento com exercício outdoor.

Em um estudo feito por Weng e Chiang (2014) é sugerido associação entre sessões de treinamento com exercício indoor e outdoor para melhoria de saúde; já os outros estudos não apontam diretamente para a análise das melhorias fisiológicas em treinamentos com exercícios indoor ou outdoor (Loureiro e Veloso, 2014; Pasanen et al., 2014; Rogerson et al., 2016; Fuegen e Breitenbecher, 2018).

\section{Discussão}

O exercício físico regular tem se constituído em uma ferramenta importante de saúde e qualidade de vida, incluindo neste campo de reflexão científica, os aspectos de bem-estar subjetivo e de conectividade com a natureza possibilitando melhorias no Índice de Desenvolvimento Humano (IDH), que é uma medida da averiguação da qualidade de vida da população, além do exercício físico regular ser uma forma de prevenir os danos do sedentarismo, que é um dos agravantes de saúde pública atual.

Nesta revisão sistemática, os resultados das pesquisas apontam na sua maioria para melhoria de indicadores de bem-estar subjetivo, conectividade com a natureza e exercício outdoor (Loureiro e Veloso, 2014; Pasanen et al., 2014; Puett et al., 2014; Weng e Chiang, 2014; Calogiuri et al., 2015; Wood et al., 2015; Rogerson et al., 2016; Krinski et al., 2017; Niedermeier et al., 2017; Fuegen e Breitenbecher, 2018), demonstrando associação direta no aumento do foco, atenção, afetos positivos, sensação de prazer, humor, energia, adesão ao exercício e diminuição de fadiga, com dados significativos em todos estes estudos, exceto em um estudo realizado por Matsouka et al. (2005) que mostra resultados similares entre treinamento com exercício indoor/outdoor e em outro estudo de Lacharité-Lemieux e Dionne (2016) que não traz análise de dados mais efetivos sobre bem-estar subjetivo. Isso demonstra um importante achado de que o exercício ao ar livre pode ser incrementado cada vez mais nas práticas de exercícios físicos regulares da população.

Ainda em termos de bem-estar, é importante destacar que os aspectos psicoemocionais vistos nos estudos da revisão sistemática apontam claramente para ganhos mais significativos na prática de exercício outdoor que a prática de exercícios indoor, indicando que os praticantes de exercícios outdoor apresentaram melhorias estatisticamente significativas nos afetos positivos, na diminuição dos afetos negativos, na melhoria do bem estar psicológico (Matsouka et al., 2005; Loureiro e Veloso, 2014; Weng e Chiang, 2014), na melhoria no humor (Calogiuri et al., 2015; Niedermeier et al., 2017; Fuegen e Breitenbecher, 2018) e na melhoria na atenção (Pasanen et al., 2014; Rogerson et al., 2016; Fuegen e Breitenbecher, 2018).

Outro aspecto observado foi que o exercício outdoor tem uma ligação com o controle do estresse, com melhor perspectiva de vida e melhor percepção de saúde com uma associação maior para populações mais ativas, enquanto que $o$ exercício físico indoor pode ser mais associados com as populações pouco ativas (Weng e Chiang, 2014)

Em se tratando da análise de conectividade com o meio ambiente todos os estudos (Matsouka et al., 2005; Loureiro e Veloso, 2014; Pasanen et al., 2014; Puett et al., 2014; Weng e Chiang, 2014;Calogiuri et al., 2015; Wood et al., 2015;Rogerson et al., 2016; Lacharité- 
Lemieux e Dionne, 2016; Krinski et al., 2017; Niedermeier et al., 2017; Fuegen e Breitenbecher, 2018; Legrand et al., 2018) apontam para melhorias na qualidade de vida na prática de exercício regular ao ar livre, exceto um estudo realizada por Legrand et al. (2018) que não demonstra diferenças significativas de melhoria entre treinamentos indoor e outdoor, no tocante a qualidade de vida e saúde. Dentre as melhorias advindas na prática de exercício ao ar livre, um estudo feito por Loureiro e Veloso (2014) mostrou que a conectividade com a natureza é um preditor de bem-estar subjetivo e saúde geral, sendo um achado também indireto nos outros estudos (Matsouka et al., 2005; Pasanen et al., 2014; Puett et al., 2014; Weng e Chiang, 2014; Calogiuri et al., 2015; Wood et al., 2015; Rogerson et al., 2016; LacharitéLemieux e Dionne, 2016; Krinski et al., 2017; Niedermeier et al., 2017; Fuegen e Breitenbecher, 2018), exceção feita apenas em um estudo de Legrand et al. (2018) que não achou diferenças significativas entre treinamentos com exercícios indoor e outdoor, como já foi citado anteriormente.

Nesta revisão sistemática (Matsouka et al., 2005; Loureiro e Veloso, 2014; Pasanen et al., 2014; Puett et al., 2014; Weng e Chiang, 2014;Calogiuri et al., 2015; Wood et al., 2015; Rogerson et al., 2016; Lacharité-Lemieux e Dionne, 2016; Krinski et al., 2017; Niedermeier et al., 2017; Fuegen e Breitenbecher, 2018; Legrand et al., 2018) observou-se que dentre os treinamentos com exercícios ao ar livre as práticas corporais mais comuns foram a caminhada, trilhas, voleibol, ciclismo e tênis, fato esse comprovado em um aumento visível destes praticantes de exercícios regulares nos ambientes urbanos e rurais do Brasil e do mundo de uma forma geral.

Outra constatação interessante foi $\mathrm{o}$ fato de em alguns estudos as pessoas que praticam exercício ao ar livre demonstraram ter maior aderência à prática regular com esta atividade, demonstrando assim um estimulo maior nos praticantes de exercícios ao ar livre em futuras intenções de voltar a realizar esta atividade novamente (Matsouka et al., 2005; Rogerson et al., 2016; Lacharité-Lemieux e Dionne, 2016; Krinski et al., 2017).

Já em relação ao aspecto fisiológico os estudos da revisão apontam resultados similares de melhorias fisiológicas com a prática do exercício físico regular, tanto com o acréscimo de treinamentos com o exercício outdoor quanto treinamentos com o exercício indoor. Na presente revisão sistemática isso pode ser contatado nos dados encontrados em algumas pesquisas que mostram resultados próximos ou similares de melhorias da frequência cardíaca e da pressão arterial (Puett et al., 2014; Weng e Chiang, 2014; Wood et al., 2015), composição corporal, índice de massa corporal e $\mathrm{VO}_{2} \max$ (Calogiuri et al., 2015; Lacharité-Lemieux e Dionne, 2016; Krinski et al., 2017), demonstrando assim não ter uma diferença estatisticamente significativa entre as duas formas de treinamento (outdoor e indoor).

\section{Conclusões}

Os treze estudos revisados apresentaram informações de suma importância sobre a relação entre exercício físico, bem-estar e conectividade com a natureza analisados na literatura nacional e internacional, sendo nesta revisão evidenciada a existência de poucos estudos sobre este tema, especialmente no contexto brasileiro, demonstrando assim a necessidade de futuras investigações que possam corroborar com os estudos já realizados em outros países.

Diante do exposto, as evidências científicas da literatura disponíveis nesta revisão envolvendo a relação entre exercício outdoor e indoor já apontam que os exercícios outdoor apresentaram dados estatisticamente significativos de melhoria e ganhos nos aspectos 
psicoemocionais, como bem-estar, humor, além de maior conectividade com a natureza, no entanto em termos fisiológicos os resultados comparativos entre exercício outdoor e indoor foram similares na melhoria da saúde geral, na composição corporal, $\quad \mathrm{VO}_{2}$ max, frequência cardíaca e pressão arterial, não mostrando assim diferenças estatisticamente significativas entre as duas categorias de prática de exercício neste aspecto, necessitado assim de pesquisas que demonstrem dados mais consistentes em nossa realidade.

A partir desta síntese, espera-se que novos estudos sejam realizados ampliando mais as evidências científicas sobre as melhorias já demonstradas, auxiliando assim o planejamento e a avaliação de intervenções com exercícios físicos outdoor para a promoção de saúde e qualidade de vida.

\section{Agradecimentos}

Aos pesquisadores participantes da pesquisa, sem os quais não seria possível viabilizar este estudo. Agradecemos também ao apoio logísitico do Programa de Pós-Graduação em Desenvolvimento e Meio Ambiente/UFPB e do Laboratório de Ecologia Comportamental e Psicobiologia (LECOPSI/UFPB).

\section{Conflito de interesses}

Os autores declaram não haver conflito de interesses.

\section{Referências}

Abrantes, M. M.; Lamounier, J. A.; Colosimo E. Comparison of body mass index values proposed by Cole et al. (2000) and Must et al. (1991) for identifying obese children with weight-for-height index recommended by the World Health Organization. Public Health Nutrition, v. 6, n. 3, p. 307-311, 2003. https://doi.org/10.1079/PHN2002426

Albuquerque, A.S.; Tróccoli, B. T. Desenvolvimento de uma escala de bem-estar subjetivo. Psicologia: Teoria e Pesquisa, v. 20, n. 2, p. 153-164, 2004. https://doi.org/ 10.1590/S0102-37722004000200008

Anjos, L. A. Índice de massa corporal (massa corporal estatura) como indicador do estado nutricional de adultos: revisão da literatura. Revista de Saúde Pública, v. 26, n. 6, p. 431436, 1992. https://doi.org/10.1590/S003489101992000600009

Blair, S. N. C. H. M. Research lecture physical activity fitness and health. Research Quarterly for Exercise and Sport, v. 64, n. 4, p. 365-376, 1993. https://doi.org/10.1080/ 02701367.2009.10599610

Bowling, A. What things are important in people's lives? A survey of the public's judgements to inform scales of health related quality of life. Social Science \& Medicine, v. 41, n. 10, p. 1447-1462, 1995. https://doi.org/10.1016/0277-9536(95) 00113-L

Browne, J. P. ; O’Boyle, C. A. ; Mcgee, H. M.; Joyce, C. R.; McDonald, N. J.; O'Malley, K. Individual quality of life in the healthy elderly. Quality of Life Research, v.3, n. 4, p. 235-244, 1994. https://doi.org/10.1007/ BF00434897

Bruhns, H. T. Lazer e meio ambiente: corpos buscando o verde e a aventura. Revista Brasileira de Ciências do Esporte, v.18, n. 2, p. 86-91, 1997.

Calogiuri, G.; Nordtug, H.; Weydahl, A. The potential of using exercise in nature as an intervention to enhance exercise behavior: results from a pilot study. Percept Motor Skills, v. 121, n. 2, p.350-370, 2015. https://doi.org/10.2466/06.PMS.121c17x0

Carvalho, Y. M. Atividade física e saúde: onde está e quem é o "sujeito" da relação? Revista Brasileira de Ciências do Esporte, v. 22, n. 2, p. 9-21, 2001.

Chao, C. H. N. Relação homem/natureza e o lazer como uma possibilidade de sensibilização de questão ambiental. Revista Motrivivência, v. 16, n. 22, p. 207-220, 2004.

Diener, E.; Napa Scollon, C.; Lucas, R. E. The evolving concept of subjective well-being: The multifaceted nature of happiness. In: Diener E. (Eds.). Assessing well-being. Dordrecht: Springer, 2009. (Social indicators research series; 39). https://doi.org/ 10.1007/978-90-481-2354-4_4 
Diener, E.; Suh, E.; Oishi, S. Recent findings on subjective well-being. Indian Journal of Clinical Psychology, v. 24, n. 1, p. 25-41, 1997.

Diener, E.; Suh, E. M.; Lucas, R. E.; Smith, H. L. Subjective well-being: Three decades of progress. Psychological Bulletin, v. 125, n. 2, p. 276-302, 1999. https://doi.org/ 10.1037/0033-2909.125.2.276

Farquhar, M. Elderly people's definitions of quality of life. Social Science \& Medicine, v. $41, \quad$ n. $10, \quad$ p. $1439-1446,1995$. https://doi.org/10.1016/0277-9536(95) 00117-P

Fleck, M. P. A. O instrumento de avaliação de qualidade de vida da Organização Mundial da Saúde (WHOQOL-100): características e perspectivas. Ciência \& Saúde Coletiva, v. 5, n. 1 , p. 33-38, 2000. https://doi.org/ 10.1590/S1413-81232000000100004

Florindo, A. A.; Salvador, E. P.; Reis, R. S.; Guimarães, V. V. Percepção do ambiente e prática de atividade física em adultos residentes em região de baixo nível socioeconômico. Revista de Saúde Pública, v. 45 , n. 2, p. 302-310, 2011. https://doi.org/ 10.1590/S0034-89102011000200009

Freitas, C. M. M.; Santiago, M. S.; Viana, A. T.; Leão, A. C.; Freyre, C. Aspectos motivacionais que influenciam a adesão e manutenção de idosos a programas de exercícios físicos. Revista Brasileira de Cineantropometria \& Desempenho Humano, v. 9, n. 1, p. 92-100, 2007.

Fuegen, K.; Breitenbecher, K. H. Walking and being outdoors in nature increase positive affect and energy. Ecopsychology, v. 10, n. 1, p. 14-25, 2018. https://doi.org/10.1089/ eco.2017.0036

Galvão, T. F.; Pansani, T. S. A.; Harrad, D. Principais itens para relatar revisões sistemáticas e meta-analises: a recomendação PRISMA. Epidemiologia e Serviços de Saúde, $\quad$ v. 24, $\quad$ n. 2, p. 335-342, 2015. https://doi.org/10.5123/S1679-49742 015000200017

Gill, T.M.; Feinstein, A. R. A critical appraisal of the quality of life measurements. Journal of the American Medical Association, v. 272, n. 8, p. 619-626, 1994.

Haskell, W. L.; Lee, I. M.; Pate, R. R.; Powell, K.; Blair, S.; Franklin, B.; Macera, C.; Heath, G.;
Thompson, P.; Bauman, A. Physical activity and public health: Updated recommendation for adults from the American College of Sports Medicine and the American Heart Association. Medicine \& Science in Sports \& Exercise, v. 116, n. 9, p. 1081-1093, 2007. https://doi.org/10.1249/mss.0b013e318061 $6 \mathrm{~b} 27$

Krinski, K.; Machado, D. G. S.; Lirani, L. S.; DaSilva, S. G.; Costa, E. C.; Hardcastle, S.J., Elsangedy, H. M. Let's walk outdoors! Self-paced walking outdoors improves future intention to exercise in women with obesity. Journal of Sport and Exercise Psychology, v. 39, n. 2, p 145-157, 2017.

Lacharité-Lemieux, M.; Dionne, I. J. Physiological responses to indoor versus outdoor training in postmenopausal women. Journal of Aging and Physical Activity, v. 24, n. 2, 275-283, 2016. https://doi.org/ 10.1123/japa.2015-0019

Legrand, F. D.; Race, M.; Herring, M. P. Acute effects of outdoor and indoor exercise on feelings of energy and fatigue in people with depressive symptoms. Journal of Environmental Psychology, v. 56, n. 4, p. 91-96, 2018. https://doi.org/10.1016/ j.jenvp.2018.03.005

Lopes, F. J. G.; Alterthum, C. C. Caminhar em busca da qualidade de vida. Revista Brasileira de Ciências do Esporte, v. 21, n. 1, p. 861-866, 1999.

Loureiro, A.; Veloso, S. Outdoor exercise, well-being and connectedness to nature. Psico, v. 45, n. 3, p. 299-304, 2014.

Marinho, A.; Inácio, H. L. D. Educação Física, Meio ambiente e Aventura. Um percurso por vias instigantes. Revista Brasileira de Ciências do Esporte, v. 28, n. 3, p. 55-70, 2007.

Marinho, A. Lazer, natureza e aventura: compartilhando emoções e compromissos. Revista Brasileira de Ciências do Esporte, v. 22, n. 2, p. 143-153, 2001.

Marselle, M. R.; Irvine, K. N.; Warber, S. Examining group walks in nature and multiple aspects of well-being: a large scale study. Ecopsychology, v. 6, n. 3, p. 134-148, 2014. http://doi.org/10.1089/eco.2014.0027

Matsouka, O. ; Kabitsis, C. ; Harahousou, Y. Trigonis, I. Mood alterations following an indoor and outdoor exercise program in 
healthy elderly women. Perceptual and Motor Skills, v. 100, n. 3, p. 707-715, 2005. http://doi.org/10.2466/PMS.100.3.707-715

Milanezi, J. Z; Nascimento Júnior, A. F.; Gonçalves, A. Expectativa de espaço e lazer dos moradores do Bairro Jardim Bela Vista, como subsídios para um programa de atividades físicas no Município de Bauru-SP. Revista Brasileira de Ciências do Esporte, v. 18, n. 2, 92-97, 1997.

Minayo, M. C. S.; Hartz, Z. M. A.; Buss, P. M. Qualidade de vida e saúde: um debate necessário. Ciências \& Saúde Coletiva, v. 5, n. 1, p. 7-18, 2000. https://doi.org/10.1590/ S1413-81232000000100002

Must, A.; Dallal, G. E.; Dietz, W. H. Reference data for obesity: $85^{\text {th }}$ and $95^{\text {th }}$ percentiles of body mass index (wt/ht2) and triceps skinfold thickness. American Journal of Clinical Nutrition, v. 53, n. 4, p. 839-846, 1991. https://doi.org/10.1093/ajcn/53.4. 839

Nahas, M. V.; Barros, M. V. G.; Francalacci, V. 0 pentáculo do bem-estar- Base conceitual para avaliação do estilo de vida de indivíduos ou grupos. Revista Brasileira de Atividade Fisica \& Saúde, v. 5, n. 2, p. 48- 59, 2000. https://doi.org/10.12820/rbafs.v.5n2p48-59

Nahas, M. V. Esporte e qualidade de vida. Revista da Associação dos Professores de Educação Física de São Paulo, v. 12, n. 2, p. 61-65, 1997.

Niedermeier, M.; Einwanger, J.; Hartl, A.; Kopp, M. Affective responses in mountain hiking: A randomized crossover trial focusing on differences between indoor and outdoor activity. Plos ONE, v. 12, n. 5, p. 1-17, 2017. https://doi.org/10.1371/journal.pone.01777 19

Pasanen, T. P; Tyrväinen, L.; Korpela, K. M. The Relationship between Perceived Health and Physical Activity Indoors, Outdoors in Built Environments and Outdoors in Nature. Applied Psychology: Health and WellBeing, v. 6, n. 3, p. 324-346, 2014. https://doi.org/10.1111/aphw.12031

Piko, B. F.; Keresztes, N. Physical activity, psychosocial health, and life goals among youth. Journal of Community Health, v. 31, n. 2 , p. $136-145,2006$. https://doi.org/ 10.1007/s10900-005-9004-2
Puett, R.; Teas, J.; España-Romero, V.; Artero, E. G.; Lee, D. C.; Baruth, M.; Sui, X.; MontresorLópez, J.; Blair, S. N. Physical Activity: Does Environment Make a Difference for Tension, Stress, Emotional Outlook, and Perceptions of Health Status? Journal of Physical Activity \& Health, v. 11, n. 8, p. 1503-1511, 2014. https://doi.org/10.1123/jpah.2012-0375

Rogerson, M.; Gladwell, V.F.; Gallagher, D. J.; Barton, J. L. Influences of green outdoors versus indoors environmental settings on psychological and social outcomes of controlled exercise. International Journal of Environmental Research and Public Health, v. 13, n. 4, p. 363-378, 2016. https://doi.org/10.3390/ijerph13040363

Ryff, C. D.; Keyes, C. L. M. The structure of psychological well-being revisited. Journal of Personality and Social Psychology, v. 69, n. 4, p. 719-727, 1995.

Sampaio, R. F.; Mancine, M. C. Estudos de revisão sistemática: um guia para síntese criteriosa da evidência científica. Revista Brasileira de Fisioterapia, v. 11, n. 1, p. 83-89, 2007. https://doi.org/10.1590/ S1413-35552007000100013

Seligman, M. E. P.; Csikszentmihalyi, M. Positive psychology: An introduction. American Psychologist, v. 55, n. 1, p.5-14, $2000 . \quad$ https://doi.org/10.1037/0003066X.55.1.5

Silva, I. J. O.; Alexandre, M. G.; Ravagnani, F. C. P.; Silva, J. V. P.; Coelho-Ravagnani, C. F. Atividade física: espaços e condições ambientais para sua prática em uma capital brasileira. Revista Brasileira de Ciência e Movimento, v. 22, n. 3, p.53-62, 2014.

Siqueira, M. M. M.; Padovan, V. A. R. Bases teóricas de bem-estar subjetivo, bem-estar psicológico e bem-estar no trabalho. Psicologia: Teoria e Pesquisa, v. 24, n. 2, p. 201-209, 2008. https://doi.org/10.1590/ S0102-37722008000200010

Tahara, A. K.; Carnicelli Filho, S.; Schwartz, G. M. Meio ambiente e atividades de aventura: significados de participação. Motriz: Revista de Educação Física, v. 12 , n. 1, p. 59-64, 2006.

Timperio, A.; Ball, K.; Salmon, J.; Roberts, R.; Giles-Corti, B.; Simmons, D.; Baur, L. A.; Crawford, D. Personal, Family, Social, and 
Environmental Correlates of Active Commuting to School. American Journal of Preventive Medicine, v. 30 , n. 1, p. 45-51, 2006. https://doi.org/10.1016/j.amepre. 2005.08.047

Weng, P. Y.; Chiang, Y. C. Psychological Restoration through Indoor and Outdoor Leisure Activities. Journal of Leisure Research, v. 46, n. 2, p. 203-217, 2014. https://doi.org/10.1080/00222216.2014.11 950320

Wood, C. J.; Pretty, J.; Griffin, M. A casecontrol study of the health and well-being benefits of allotment gardening. Journal of Public Health, v. 38, n. 3, p. 336-344, 2016.

CC Informação da Licença: Este é um artigo Open Access distribuído sob os termos da Licença Creative Commons Attribution, que permite uso irrestrito, distribuição e reprodução em qualquer meio, desde que a obra original seja devidamente citada. 